\title{
Rethinking construction: the Generic Design and Construction Process Protocol
}

\author{
MICHAIL KAGIOGLOU, RACHEL COOPER, GHASSAN AOUAD* \& MARTIN SEXTON* \\ Research Institute for Design and Manufacture, University of Salford, Centenary Building, Peru Street, Salford, M3 6EQ \\ and ${ }^{\star}$ Research Centre for the Built and Human Environment, University of Salford, Salford, M5 4WT, UK
}

\begin{abstract}
The complexity of construction projects and the fragmentation of the construction industry undertaking those projects has effectively resulted in linear, uncoordinated and highly variable project processes in the UK construction sector. Research undertaken at the University of Salford resulted in the development of an improved project process, the Process Protocol, which considers the whole lifecycle of a construction project whilst integrating its participants under a common framework. The Process Protocol identifies the various phases of a construction project with particular emphasis on
\end{abstract}

what is described in the manufacturing industry as the 'fuzzy front end'. The participants in the process are described in terms of the activities that need to be undertaken in order to achieve a successful project and process execution. In addition, the decision-making mechanisms, from a client perspective, are illustrated and the foundations for a learning organization/industry are facilitated within a consistent Process Protocol.

Keywords activity zones, design and construction, project process, process map, Process Protocol, stage gate

\section{INTRODUCTION}

The construction industry in the UK is plagued with a number of problems, which have not disappeared in the last few decades. Those problems have been illustrated by several government reports, including Simon (1944) and more recently the Latham (1994) and Egan (1998) reports. The findings are familiar practice to the majority of the industry, which is still striving towards improvements in a number of areas, but apparently with little success. There are three main areas for consideration:

1. development of a solution: including consultation with the client and development of product specifications and design;

2. implementation of the solution: the construction or refurbishment of the facility that will satisfy the client needs and those of the project participants; and

3. the project process: the roadmap or framework that is used for undertaking the project activities and that delivers value to the supply chain parties.

It is, however, almost impossible to consider any of the aforementioned areas in isolation as they are all interdependent and suboptimization does not guarantee project success.

The Latham (1994) report reaffirmed the conclusions of all previous studies on the subject. The report focused on the fragmented nature of the industry as a major contributing factor to the poor communication between all parties working on a construction project. The main outcome and recommendation of the Latham report was its call for significant cost savings by the utilization and formulation of effective construction processes, which will in turn lead to increased performance. The recommendations of this report were reaffirmed in a recent report by Egan (1998), which reported to the deputy Prime Minister John Prescott on the scope for improving the quality and efficiency of UK construction. This report identified the following five key drivers of change that need to set the agenda for the construction industry at large:

1. committed leadership;

2. focus on the customer;

3. integrated processes and teams;

4. quality-driven agenda; and

5. commitment to people.

Within the focus for integrated processes and teams, four key elements were identified: product development; project implementation; partnering the supply chain; and production of components. Furthermore, the Egan (1998) report called for annual reductions of $10 \%$ in construction cost and time and an annual reduction of $20 \%$ in project defects. This total performance improvement of $30 \%$ requires significant improvements in the way that the construction process is 
enacted. It will require a significant reengineering of the construction process and the subprocesses involved in undertaking construction works.

\section{LEARNING FROM MANUFACTURING}

The findings and recommendations of Latham and Egan relate to the state of the construction industry at the present time and offer some clues as to how some of the problems might be overcome by transferring established practices from the manufacturing industry. Indeed, this view has been expressed by a number of researchers and practitioners (Cooper et al. 1998; Kagioglou et al. 1998a; Koskela 1992). However, the authors believe that the transfer of knowledge and practices from manufacturing into the construction industry should be treated with caution for a number of reasons. First, the level of maturity of both processes and practices is quite different, with manufacturing having the 'lead'. Second, the structure of the industries and of the organization of project personnel can be different, in that construction relies heavily on Temporary Multi-organizations (TMOs) whereas manufacturing normally operates within long-term partnership arrangements. Finally, comparison between the processes and the practices of both industries must be made by considering the levels in which they exist, i.e. strategic, managerial and operational. Therefore, clarification of process levels can have an important influence on the management of those processes.

There are two main areas of manufacturing that construction can benefit from (Kagioglou et al. 1998b): the project process or New Product Development (NPD), as it is known; and the operational/production processes. The first relates very closely, both in terms of nature and content, to the design and construction process. As such, it considers the development of a solution (usually a tangible product) from a need identified in the market place or internally within an organization to the implementation of that solution and the eventual withdrawal of the product. This is achieved by organizing the activities that need to take place in a number of phases, which are made distinct by the determination of review points between the phases. This is very similar to the enactment of a construction project, the difference being that the distinction between the phases is usually determined by the entry of the different parties/ functions, i.e. architects, contractors etc., to the process. The second area is related to the way in which the production of a product, including material flow, process design and resources planning, is undertaken. Indeed, a number of very effective philosophies and practices such as Just in Time (JIT), lean production and others have a legacy of optimized production in the manufacturing sector. JIT aims to improve production by utilizing the internal and external supply chains in terms of people and material flow. The similarities of JIT, for example, in the construction sector can be illustrated by listing a number of the benefits, amongst many, that are offered by its successful implementation:

- reduced time-scales by optimizing material flow;

- reduced waste of both materials and processing time by optimizing the manufacturing processes; and

- supply chain integration by utilizing effective partnerships.

The first two benefits can be realized in the construction industry (see, for example, Koskela 1992) perhaps more readily than the third one, which requires a significant reorganization and mind-shift of the litigation-driven industry.

This paper concentrates on the lessons that can be learned from the NPD/project process of manufacturing, and reference to it is made throughout the description of the Generic Design and Construction Process Protocol (GDCPP).

\section{THE GENERIC DESIGN AND CONSTRUCTION PROCESS (GDCPP) PROTOCOL}

This section presents a description of the research methods and the main findings of an Engineering and Physical Sciences Research Council (EPSRC) funded project under the Innovative Manufacturing Initiative (IMI) 'Construction as a Manufacturing Process' initiative. The project brought together a number of companies, representing the construction supply chain, and the University of Salford's research expertise to produce the 'Generic Design and Construction Process Protocol'.

The main aims of the project were to:

- develop an improved design and construction Process Protocol by analysing the current practices in the construction industry and drawing comparisons with similar practices in the manufacturing industry; and

- identify the Information Technology (IT) requirements needed to support the Process Protocol and 
develop demonstrator models (the results of the IT investigation are presented elsewhere).

This project involved contractors, clients, IT specialists and the supply chain coming together with academia through a government-stimulated research programme to develop an improved design and construction process. This also enabled the production of a solution that is devised, owned and satisfactory to all parties. The GDCPP project and this paper relate most appropriately to the research programme under which it is funded ('Construction as a Manufacturing Process'), and the nature of Latham's and Egan's philosophical approaches to achieving the resolution of client dissatisfaction, construction industry dissatisfaction and supply chain problems.

\section{Concept of Process Protocol}

The concept of the Process Protocol was based on a number of issues and deficiencies of current practices in the construction industry. This enabled the identification of areas for improvement by examining and comparing best practice in manufacturing project processes. To this end, a need was identified for a model that is capable of representing the diverse interests of all the parties involved in the construction process or that is able to provide a complete overview. In addition, the design and construction operations need to form part of a common process (model) best controlled by an integrated system. This has been achieved successfully in manufacturing and other industries through process modelling and the reengineering of those processes (Rosenau 1996). In order for construction to utilize such a model/process approach, there is a need for a coherent and explicit set of process-related principles, a new process paradigm, which can be managed and reviewed across the breadth and depth of the industry and which focuses on changing and systematizing the strategic management of the potentially common management processes in construction whilst accommodating the fragmentary production idiosyncrasies. In addition, it is appreciated that there will be no best way for all circumstances; however, a generic and adaptable set of principles will allow the consistent application of principles in a repeatable form. Finally, a philosophy of early entry into the process of the key functions needs to be incorporated, with the emphasis of the effort on design and planning to minimize both error and reworking during construction.

\section{RESEARCH METHODOLOGY}

The research team endeavoured to use a methodology that was sympathetic to the issues being investigated: in effect to '... suit the method to the problem, and not the problem to the method' (Linstone 1978). Furthermore, it was appreciated that different types of issues would be encountered during the development of the Process Protocol, and that these often disparate issues would be best served by a variety of research methods. To provide the necessary contingency-based, but integrated, research methodology to accommodate these differing demands in a coherent and consistent way, an overall research model was developed, as shown in Fig. 1.

The outer ring of Fig. 1 represents the unifying research philosophy, which guides and energizes the inner research approaches and research techniques. Research approaches consist of the dominant theory generation and testing methods. Research techniques comprise data collection tools.

The nesting of the model's elements generated a framework, which provided the research team with an interactive portfolio of approaches and techniques that benefited from meta-level direction and cohesion. Each of the model's elements will be briefly discussed.

\section{Actor-based research philosophy}

The overarching research philosophy used in the GDCPP project was the preunderstanding-understanding hermeneutic spiral (Odman 1985), grounded in actor

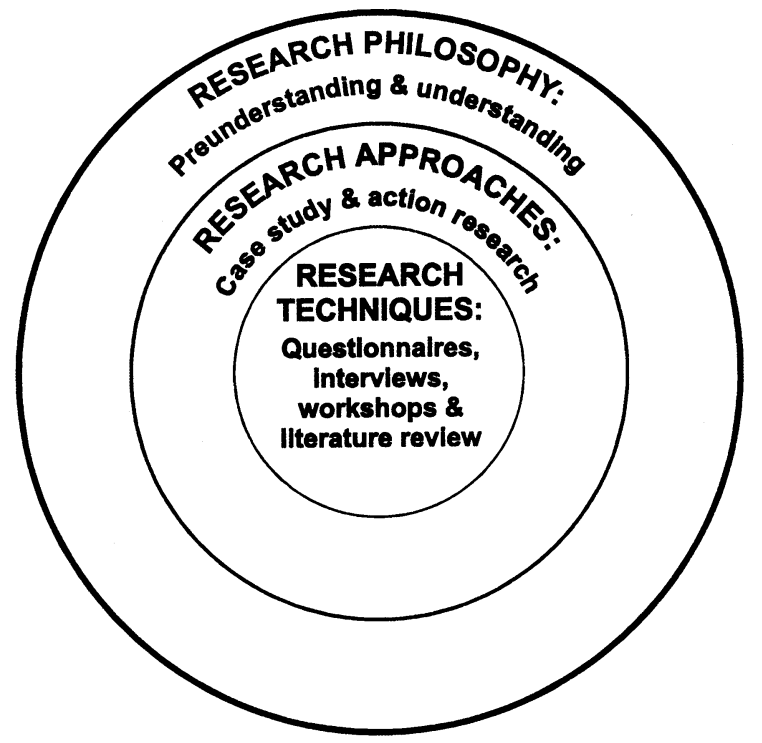

Figure 1 Overall GDCPP research model. 


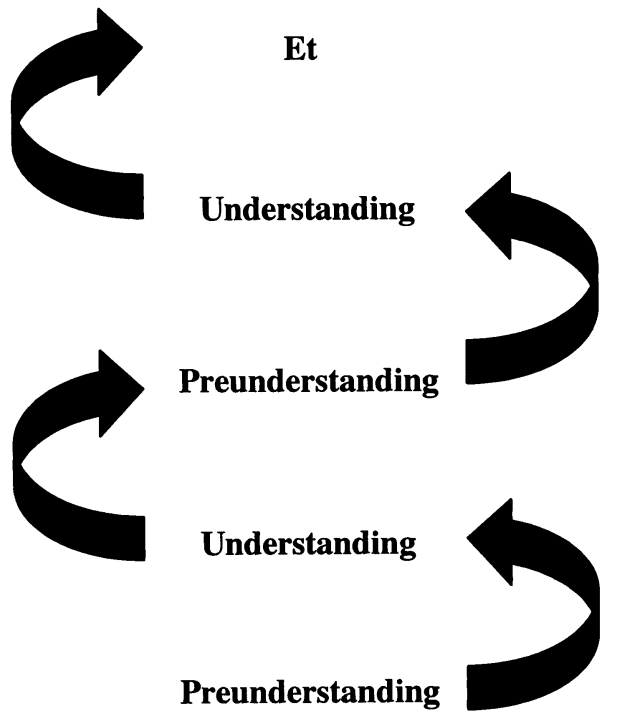

Figure 2 Hermeneutic learning spiral.

research philosophy (see, for example, Berger \& Luckmann 1966; Sandywell 1975). The spiral, shown in Fig. 2, depicts research as an iterative process whereby the industrial partners' and the University of Salford research team's a priori knowledge, insights and experience form the preunderstanding, or common language, to inform the subsequent stage of understanding, which furthers the development of the Process Protocol.

This understanding, in turn, is the basis for the preunderstanding of the next stage of development, and so on.

The main conduit for the translation and elevation of preunderstanding into understanding were workshops, although crucial preunderstanding/understanding was transferred and developed through an ongoing dialogue both prior to and between the workshops. The workshops and intervening dialogue improved both individual and collective learning throughout the project team.

\section{Research approaches}

\section{Case studies}

A traditional case study approach was used, with University of Salford researchers entering industrial partners' organizations openly in the role of investigators, with the express purpose of learning more about their activities with respect to the design and construction processes being practised. Three case studies were undertaken - one in a manufacturing organization and two projects undertaken by Alfred McAlpine's Special Projects Division.

\section{Action research}

The methodology was infused with an action research/ learning dimension, in as much as the workshops not only facilitated the generation of new knowledge or understanding but also provided structured frameworks for carrying out organizational change within the boundaries of the industrial partner research team members.

The action research strand was firmly anchored to the following interactive assumptions:

- the optimal way of learning about the design and construction process was through attempting to initiate a generic Process Protocol-based change within it;

- the industrial partners who would be affected by, or instrumental in, these changes should as far as possible be involved in the workshops; and

- workshop participation would encourage industrial partner members to generatively learn as they discovered how to make sense of the Process Protocol in terms of their own language and organizational setting.

The action research model shown in Fig. 3 was implicit in the design and implementation of the workshop stages. The model channels the action research effort through an optimal flow of stages - starting with bringing the workshop team together in the centre and then moving to agreeing the scope for action at the top.

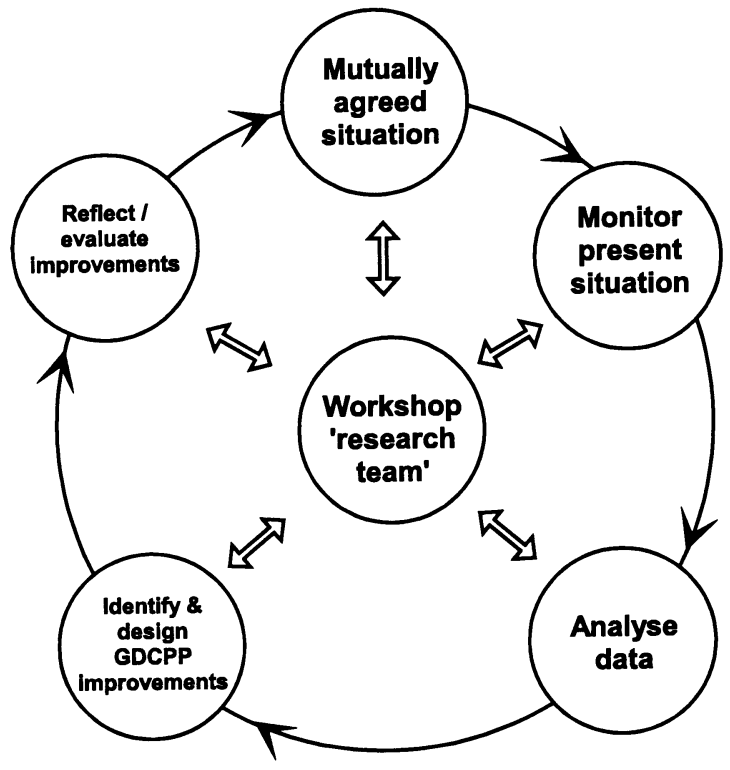

Figure 3 The action research process model. 
The process is not linear, but interactive.

\section{Techniques of research}

\section{Questionnaire surveys}

Use was made of self-completion questionnaires during the initial scoping and case study elements of the project. The questionnaires collected predominantly qualitative data from the partners in the project.

The questionnaire surveys aimed to generate factual and attitudinal information and understanding (see, for example, Ackroyd \& Hughes 1983). The factual elements of the questionnaires sought to gain information from individuals within the industrial partners firms concerning the material aspects of design and construction processes: for example, project duration time. The attitudinal aspects of the questionnaires aimed to secure data on what individuals felt about a given issue: in this case, for example, what they felt about the effectiveness of the prevailing performance review process.

\section{Workshops}

The workshops carried out during the project were central to fruitful preunderstanding and understanding progression. Each workshop had a specific task to investigate, which was set out and managed by a University of Salford co-ordinator. The workshop configuration, in effect, created a boundary-spanning team, which could tackle complex process issues by bringing together and harnessing a diverse range of expertise in a structured way.

The Workshops were designed and implemented to:

- Provide feedback such that the co-ordinator facilitated learning by providing workshop members with feedback about the consequences of issues being raised and decisions being taken.

- Stimulate questioning: the co-ordinator facilitated learning by asking questions that stimulated participants to think in new ways. For example, issues were often reframed from a manufacturing perspective and returned to the forum as a question.

- Permit modelling and validation: the co-ordinator encouraged learning and understanding through process models that participants could either adopt, modify or reject. For example, the Process Protocol map itself underwent numerous changes as a direct result of workshop debate.
- Provide support: the co-ordinator facilitated learning by providing a psychologically supportive environment, thereby creating a climate where novel issues and questions could be raised. For example, ideas were generated from a variety of construction perspectives as well as an injection of diverse theoretical views.

- Structuring: the co-ordinator facilitated learning by structuring the flow of work. For example, in one workshop, a hypothetical construction case was developed to explore process issues.

\section{Interviews}

Interviews were used throughout the research process. In total, there were 30 interviews helping the development of the Process Protocol and 30 interviews during the case study investigations. They were generally semi-structured in nature to allow it to have an overall purpose but be sufficiently flexible to explore issues as they arose during the discussion. The contents of interviews were validated by the sending of case study reports for comment to the relevant industrial partners.

\section{Approaches to modelling the process}

Given the apparent lack of commonality in the contemporary understanding of the design and construction process, an attempt was made to produce a model of the process that could be debated and subsequently refined towards a generic representation.

The initial model was developed based on existing descriptions of the design and construction process (inter alia Walker 1989; Hughes 1991), some case study data and reviews of other published models (inter alia RIBA 1980; Sanvido 1990; BAA Plc. 1995)

The Integration Definition language 0 for Function Modelling (IDEF-0) process modelling technique was adopted, initially, as the most appropriate means of representing this process. It is this technique that has been used to successfully represent processes such as Sanvido's Integrated Building Process Model (Sanvido 1990).

In developing a process model using the IDEF-0 technique, an initial step is the establishment of the activities that will comprise the model. These activities were presented for discussion in preliminary workshop sessions with the project's industrial partners. However, initial reactions to this were poor, principally because such an approach did not facilitate communication of the process, either quickly or clearly. 
Moreover, it was found that the partners, at this stage, also preferred to concentrate on the general principles of the process (see later section) rather than the detail of the activities involved.

This preference for principles was found to have a certain congruence with other models of manufacturing processes. Cooper's discussion of the evolution of the 'stage gate' models in manufacturing (Cooper 1994) and other (inter alia GPT, Fisons) industrial models demonstrate this. In such models, the graphical representation of the process conveys its inherent principles.

Furthermore, the IDEF-0 technique has been found to be effective when modelling 'as-is' processes; however, it shows limitations when the modelling of an improved 'will-be' process is attempted. In response to this, the project team has adopted a scenario-building technique whereby the participants of workshops were asked to 'act' the roles of various functions in the industry. In such, the current pitfalls of the design and construction process were identified and improvements suggested. Those improvements were further designed in a diagrammatic illustration of the process (see Fig. 6), which was easy to understand and communicate while at the same time offered a vision of the future.

\section{Literature review}

A wide literature review of primary, secondary and tertiary sources was carried out.

The following literature reviews were undertaken.

\section{Literature review of NPD in manufacturing}

NPD in manufacturing relates closely to the design and construction process. This literature review identified the development of the stage gate approach through three generations and its use in the manufacturing industry. It also identified the key principles underlying stage gate methodologies, which could be transferred to the construction sector.

\section{Literature review of process in construction}

This identified the various research groups conducting relevant work. For example, Agile construction (Bath University), Design Management (Loughborough University), Construction Business Process Reengineering CORE (Southampton University), Sanvido (Pennsylvania University) and Lean Production (VTT Finland). The review also identified some construc- tion-related process maps such as BAA, RIBA plan of work and British Property Federation (BPF).

\section{Literature review of IT support for construction}

Projects such as Architecture, Methodology and Tools for Computer-Integrated Large-Scale Engineering (ATLAS), COmputer Models for the Building INdustry in Europe (COMBINE), COnstruction Modelling and Methodologies for Intelligent information inTegration (COMMIT), Simultaneous Prototyping for An integrated Construction Environment (SPACE), Information/integration for CONstruction (ICON), Open Systems for CONstruction (OSCON) were investigated as to their relevance to the project.

These literature reviews enabled the development of the key principles underlying a process for design and construction. In conjunction with an assessment of process modelling techniques, the reviews contributed to a first model of the Process Protocol and the IT, which could support it.

\section{KEY PRINCIPLES OF THE PROCESS PROTOCOL}

As a result of the initial review of the literature and the identification of the industry's requirements through additional interviews with practitioners, six key principles are considered to provide the basis for an improved process. They are drawn heavily from the manufacturing sector, where process thinking and continuous improvement has been focused on for some 30 years. In addition, many of the principles relate to recognized problem areas in construction, where significant improvements have been called for (inter alia Banwell 1964; Latham 1994). The six principles are as follows.

\section{Whole project view}

In the construction industry, the definition of a project has traditionally been synonymous with actual construction works. As such, the pre- and postconstruction activities have been sidelined and often accelerated to reach the construction stage or to move on to the 'new job'. This has resulted in poor client requirements identification and has delayed the exposure of any potential solutions to the need to any internal and external specialists. Any contemporary attempt to either define or create a 'design and construction process' will have to cover the whole 'life' of a project from recognition of a need to the operation of 


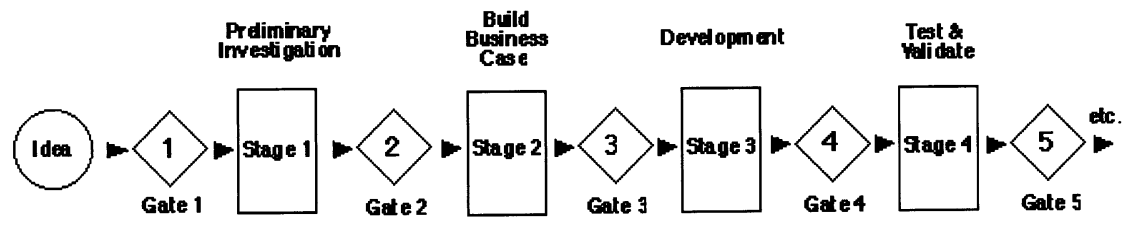

Figure 4 The 'stage gate' approach.

the finished facility and, finally, to its demolition. This approach ensures that all issues are considered from both a business and a technical point of view. Furthermore, it recognizes and emphasizes the interdependency of activities throughout the duration of a project. It is also focused at the 'front end' activities, whereby attention is paid to the identification, definition and evaluation of client requirements in order to identify suitable solutions.

\section{A consistent process}

During the review of existing models and descriptions of the design and construction process, it was quickly established that little consistency existed. In such an environment, the problems encountered by TMOs working can be compounded. Luck \& Newcombe (1996) supported this view, describing the 'role ambiguity' commonly associated with construction projects. Development of this generic Process Protocol provides the potential to establish its consistent application. Consistency of use should reduce the scope for ambiguity. This, together with the adoption of a standard approach to performance measurement, evaluation and control, should facilitate a process of continual improvement in design and construction.

\section{Progressive design fixity}

The 'stage gate' approach found in manufacturing processes (Cooper 1994) applies a consistent planning and review procedure throughout the process, as shown in Fig. 4.

Phase reviews are conducted at the end of each phase with the aim of reviewing the work executed in the phase, approving progress to the next phase, and planning the resourcing and execution of the next one. Cooper (1994), in his third generation process, saw the need for 'conditional-go' decisions at phase gates, to accommodate aspects of concurrency. This philosophy is translated in the development of the protocol's phase gates. Phase gates are classed as either soft or hard, with the 'soft gates' allowing the potential for concurrency in the process whilst ensuring that the key decision points in the process are respected, as shown in Fig. 5.
The potential benefit of this approach is fundamentally the progressive fixing and/or approval of information throughout the process. As Cooper (1994) stated, the discipline of the phase review activity improved the conventional chaotic, ad hoc approach of manufacturing to which the construction industry of today could be compared.

\section{Co-ordination}

Co-ordination is one area in which construction is traditionally perceived to perform poorly. This perception is supported by Banwell (1964) and Latham (1994), in addition to many other reviews of the industry. The need for improved co-ordination was also highlighted by the interviews with senior managers undertaken during the research project.

It is, therefore, proposed that co-ordination of the Process Protocol is undertaken, principally, by the process/change management Activity Zones (see Fig. 6). Appointed by the client, the process manager will be delegated authority to plan and co-ordinate the participants and activities of each phase throughout the process. The actions of the process manager are supported by the change manager, through whom all information related to the project is passed. In this role, the change manager acts as the official interface between both the Activity Zones in the process and, ultimately, the legacy archive.

\section{Stakeholder involvement and teamwork}

Manufacturing industries have recognized that multifunction teams, established in a development process, reduce the likelihood of costly changes and production difficulties later on in the process, by enabling design and manufacturing decisions earlier in the process.

Conventionally, many building projects comprise a team of participants assembled specifically to facilitate the development of that single project. Consequently, a complete project team rarely works together on more than one project and, as Sommerville \& Stocks (1996) argued, this can negatively affect the assembled 'team's' performance. In addition, many key contributors are identified and included too late in the process. 
Project success relies on the right people having the right information at the right time. Proactive resourcing of phases through the adoption of a 'stakeholder' view should ensure that appropriate participants (from each of the key functions) are consulted earlier in the process than is traditionally the case. This, in itself, will not eliminate the problems associated with TMO working. However, the active involvement of all participants, especially in the early phases of a project, may subsequently help to foster a team environment and encourage appropriate and timely communication and decision-making.

\section{Feedback}

In addition to the direct teamwork problems associated with TMOs, the ability to learn from experience is also hampered by the continual formation and break-up of project teams. Both success and failure can offer important lessons for the future; however, the fragmented and competitive nature of the construction industry prevents the benefits of shared best practice being utilized. The phase review process facilitates a means by which project experiences can be recorded throughout the process, thereby informing later phases and future projects. Competitive advantage will arise from how such experiences are acted upon. Shared knowledge may not automatically increase the competitiveness of companies working in construction. This Process Protocol, therefore, proposes the creation, maintenance and use of a legacy archive, which acts as a central repository or information spine (Sheath et al. 1996), for the information generated through each of the phases of the process. The subsequent increase in awareness, project to project, has the potential for reducing risk and improving performance, which, over time, may ultimately meet Latham's expectations.

\section{Process Protocol elements}

The Process Protocol model is presented in Fig. 6. Essentially, the model breaks down the design and construction process into 10 distinct phases. These are grouped into four broad stages, namely preproject, preconstruction, construction and postconstruction.

\section{Preproject stage}

The preproject phases relate to the strategic business considerations of any potential project that aims to address a client's need. Throughout these phases, the client's need is progressively defined and assessed with the aim of:

- determining the need for a construction project solution; and

- securing outline financial authority to proceed to the preconstruction phases.

In currently acknowledged models of the design and construction process (inter alia RIBA 1980; British Property Federation 1983; Hughes 1991 provides a comprehensive review) and in recently published client-focused guides (CIRIA 1995), this stage of a project is given scant consideration, when compared with the latter stages. However, the models assume that clients have already established 'the need' when approaching the construction industry. Whilst there is little evidence to suggest that this is not the case, it would seem reasonable to assume that the knowledge possessed by speculative building developers and consultants could assist any client in these early stages of a project. The problems associated with the translation of this need through the conventional briefing stage of design (O'Reilly 1987) have the potential for substantial elimination via such an approach.

\section{Preconstruction stage}

With outline financial approval obtained, the process progresses through to the preconstruction phases where the defined client's need is developed into an appropriate design solution. Like many conventional models of the design process, these phases develop the design through a logical sequence, with the aim of delivering approved production information. The phase review process, however, adds the potential for the progressive fixing of the design, together with its concurrent development, within a formal, co-ordinated framework. Progressive fixity should not be confused with 'design freeze' although, to some, this may be a desired aspect of the process. The major benefit of the fixity of design is the potential for improved communi-

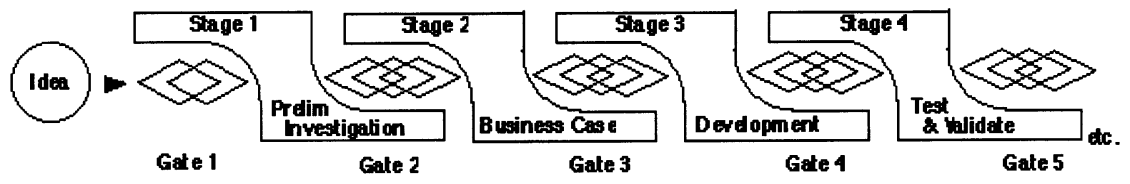

Figure 5 The 'fuzzy gate' approach. 

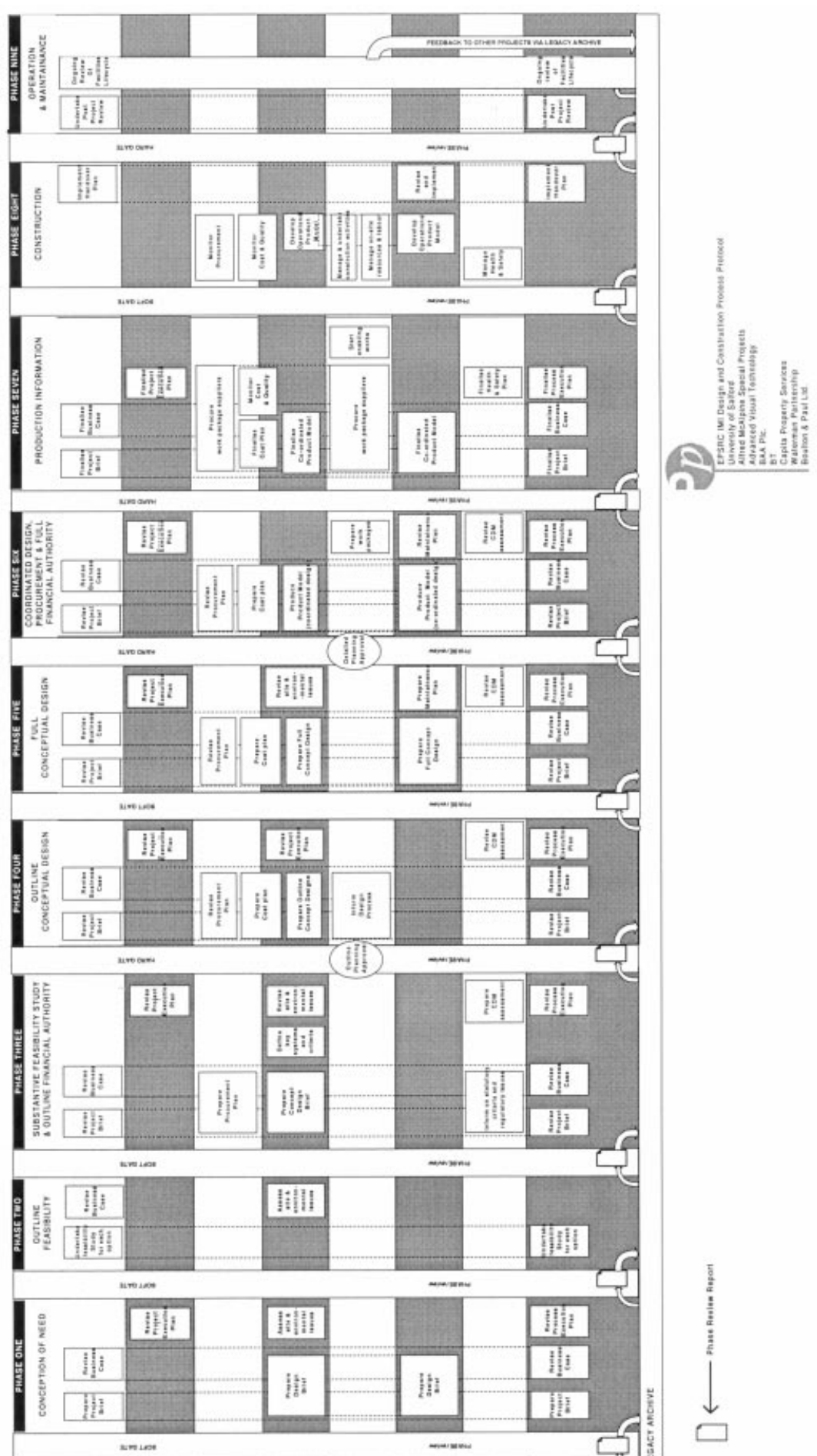

$\downarrow$
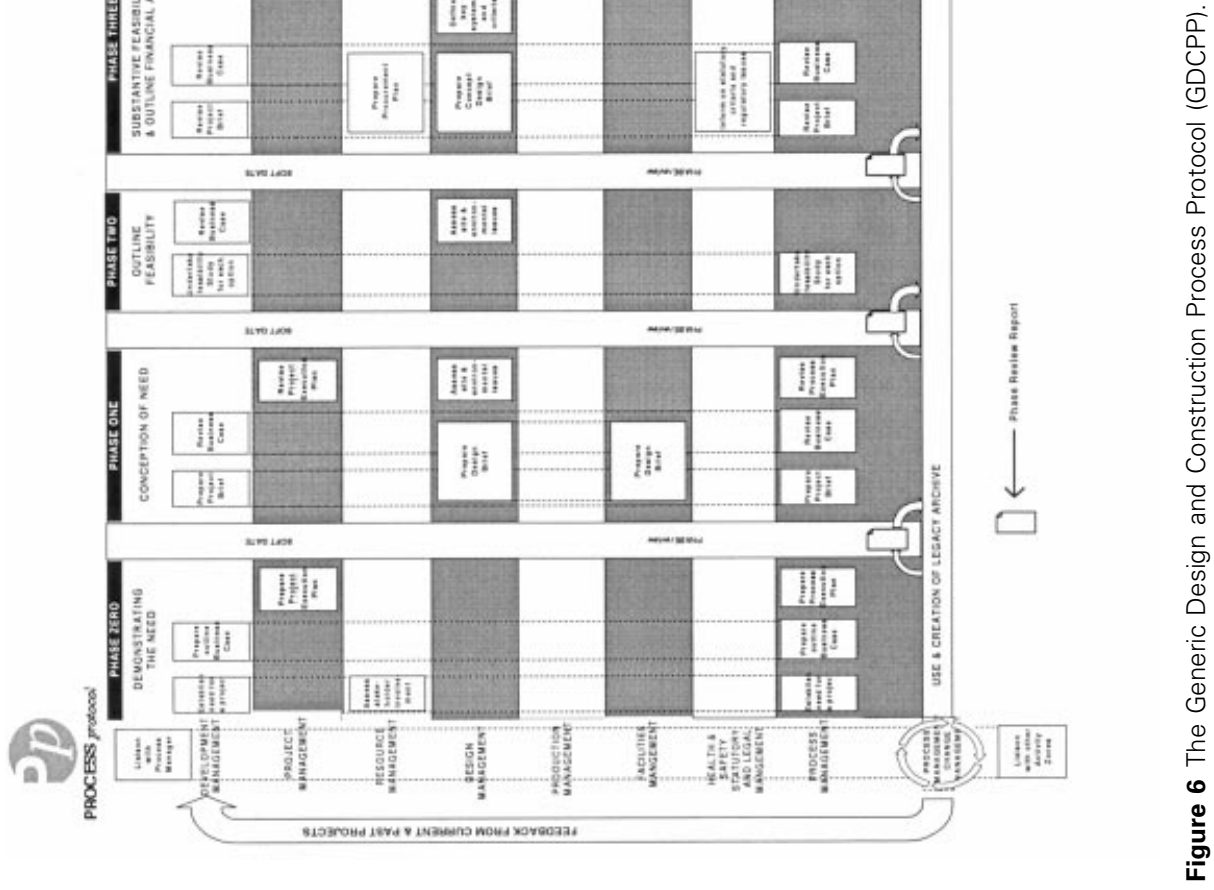
cation and co-ordination between the project's participants as they pass through each phase. Given the dynamic market conditions that influence many construction client's decisions, the need for flexibility must be addressed by the industry. At the end of these phases, the aim is to secure full financial authority to proceed. Only on such authority will the construction phase commence; this decision will be easier to make when the extent of the work and its associated risks can be readily understood.

\section{Construction stage}

The construction phase is solely concerned with the production of the project solution. It is here that the full benefits of the co-ordination and communication earlier in the process may be fully realized. Potentially, any changes in the client's requirements will be minimal, as the increased cost of change as the design progresses should be fully understood by the time on-site construction work begins.

The 'hard gate' that divides the preconstruction and construction phases should not prevent a 'work package' approach to construction and the associated delivery time benefits that this brings. As with all activities in the process, where concurrency is possible it can be accommodated. The hard and soft gates that signify phase reviews merely require that approval is granted prior to such an activity being carried out.

\section{Postcompletion/construction stage}

Upon completion of the construction phase, the Process Protocol continues into the postconstruction phases, which aim to continually monitor and manage the maintenance needs of the constructed facility. Again, the full involvement of facilities management specialists at the earlier stages of the process should make the enactment of such activities less problematic. The need for surveys of the completed property, for example, should be avoided as all records of the development of the facility should have been recorded by the project's legacy archive.

\section{The subprocesses: 'activity zones'}

The earlier involvement of the project's participants throughout the process is a significant development of the conventional approach to building. Traditionally, a construction project's participants are referred to by their professional or expert status. Ball (1996) demonstrated how this may be attributed to the inherent class relations associated with each of the professions and expert groups. As with all class distinctions, the effect that this basis for organizational structure in design and construction has is division.

A consequence of this traditional approach, by which even the more recent forms of contract procurement (design and build, management contracting etc.) are included, is the poor communication and co-ordination commonly associated with construction projects.

The participants in the Process Protocol are referred to in terms of their primary responsibilities and are represented on the $Y$-axis of the process model. It is recognized that traditionally, project to project, organizational roles and responsibilities change, resulting in ambiguity and confusion (Luck \& Newcombe 1996). By basing the enactment of the process on the primary responsibility required, the scope for confusion is potentially reduced and the potential for effective communication and co-ordination increased. The Process Protocol groups the participants in any project into 'activity zones'. These zones are not functional but rather they are multi-functional and represent structured sets of tasks and processes that guide and support the work towards a common objective (for example, to create an appropriate design solution).

A single person or firm can carry out an activity zone in small projects; however, in large and complex projects, an activity zone may consist of a complex network of people and between relevant functions and/or organizations. Because they are multi-functional, membership of the 'zones' is determined by the specific project task and/or process. For example, design management often has important input in the production management and facilities management activity zones amongst others and vice versa.

Of the activity zones associated with the model, not all will be discussed here in detail. Most of the 'zones' are self-explanatory. However, the role of the process/ change management and development management activity zones will be described, as they present a significant departure from the conventional view of the design and construction process.

\section{Process/change management}

The process/change management activity zones are essentially the interface between the development management and the other project participants. Process management has a role independent of all other activity zones. A distinction must be made between this conventional view of a project manager and the 
process management role. Process management, as the title suggests, is concerned with the enactment of the process rather than the project. Key to the success of each phase in the process is the production of project deliverables (reports and documentation associated with each phase). In this respect, the process management is responsible for facilitating and co-ordinating the participants required to produce the necessary deliverables. Acting as the development management's 'agent', it will ensure the enactment of each phase as planned, culminating with the presentation of the deliverables at each end of phase review.

The change management function is further distinct from the process management zone, as this role is solely concerned with (as its name also suggests) the management of change(s) that occur during the process. As the project becomes increasingly defined as each phase is enacted, changes (or rather updates) to the information required for the development of the project will be produced. These updates will be contained within the work required to develop the deliverable documentation associated with each phase. With respect to this, the change management activity zone facilitates the holding, review and dissemination of all this information as the project progresses.

It is within the change management function that IT potentially plays a fundamental role. Given the vast amount of information generated throughout a project's lifecycle (Aouad et al. 1994), and the need for its quick and effective dissemination, IT may offer a capable solution. However, the need for judgement and discretion, especially in the earlier strategic phases of the process, will always involve the development management's intervention and this alone is likely to prohibit the use of IT as a total solution. Aouad et al. (1998) further described the role of IT within the Process Protocol.

\section{Deliverables}

Each gate of the Process Protocol represents a decision-making point. The decisions are based primarily on documented project and process information, which are called deliverables. They are primarily compiled by project management to form the phase review report. The report includes all the deliverables (see Fig. 6) specific to the phase and as they are defined by the Process Protocol for the specific project. This phase review report forms the basis for the client body (i.e. development management) to make a decision concerning the future of the project.
The deliverables are 'live' documents, which change throughout the majority of the process. They can be in one of the following states:

- initial: preliminary information is presented;

- updated: current information is updated;

- revised: major changes/decisions will significantly alter the content and context of the deliverable; and

- finalized: the information presented is agreed and is unlikely to change throughout the duration of the project.

\section{Summary and concluding remarks}

The principles of the Process Protocol can, therefore, be summarized as a framework model that is capable of representing the diverse interests of all the parties involved in the process, which is sufficiently repeatable and definable to allow IT to be devised to support this management and information management. Therefore, a mechanism by which the systematic and consistent interfacing of the existing practices, professional practice and IT practice support tools can be facilitated. The simplicity within the protocol allows its interpretation and flexible application. This is achieved at a variety of strategic levels across a variety of scales of projects, using combinations of virtual teams and IT systems; all are based within clarity in terms of what is required from whom, when and with whose co-operation; for whom the requirements are to be delivered, for what purposes and how they will be evaluated (through the phase review board). Other principles underlying the Process Protocol were the standardization of deliverables and roles associated with achieving managing and reviewing the process and the product.

The Process Protocol is divided into a series of subphases defined as preproject, preconstruction, construction and postconstruction; within each of these major phases are subphases that can be operated concurrently or concatenate to make the process more efficient in smaller scale projects.

Novelty arises within the Process Protocol in a number of areas, in particular: the extension of the boundaries of design and construction process into the requirements capture phase of prebriefing client decision-making; the extension of the boundary of the process beyond practicable completion to allow the management of use and the learning from performance in use to improve the product and process for future projects; the creation of an explicit process management and change management role to co-ordinate the functionaries and deliverables associated with the pro- 
cess, the information that supports the functional roles and is delivered via the creation and products, and a stable platform to allow innovations in process and in products and operations to be facilitated in a co-ordinated and repeatable manner.

\section{Subprocess development}

The effective implementation of the Process Protocol will greatly depend on its ability to effectively translate the strategic to the operational level. To this end, further work (which has been initiated) is needed in examining the subprocesses (activity zone) and to produce generic maps for those subprocesses.

In such a way, the underlying principles and philosophies of the Process Protocol will form the framework for company/project-based wide adoption and effective implementation. This is confirmed by the adoption of the Process Protocol by the CRISP Process Group with the comment that sublevel process definition needs to be defined. In addition, such development will address all of the issues identified in the Construction Round Table (CRT) 'Agenda for Change'. Furthermore, a number of companies such as Alfred McAlpine, BT, Tarmac, AMEC, BNFL, $\mathrm{BAe}$, and institutions such as the International Alliance for Interoperability (IAI) Client Briefing Domain have adopted the Process Protocol as a framework and process management mechanism.

Furthermore, a generic (subprocess) process will also contribute to cultural change in terms of improved communication and process management between the fragmented groups within the construction industry.

\section{ACKNOWLEDGEMENTS}

The authors would like to acknowledge the contributions of John Hinks, Darryl Sheath, all of the partner companies and those companies and institutions who enabled the data collection. In addition, we are grateful to the Engineering and Physical Sciences Research Council (EPSRC) for their funding and support.

\section{REFERENCES}

Ackroyd, S. \& Hughes, J. (1983) Data Collection in Context. Longman, London.

Aouad, C., Brandon, P., Brown, F., Cooper, G., Ford, S., Kirkham, J., Oxman, R., Sarshar, M. \& Young, B. (1994) Integration of construction information (ICON): final reportintegrated databases for the design construction and management of construction. University of Salford.

Aouad, G., Hinks, J., Cooper, R., Sheath, D.M., Kagioglou, M. \& Sexton, M. (1998) An IT map for the Generic
Design and Construction Process Protocol. Fournal of Construction Procurement, 4, 132-151.

BAA Plc. (1995) The Project Process. BAA Plc., UK.

Ball, M. (1996) Rebuilding Construction. Routledge, London.

Banwell, H. (1964) Report of the Committee on the Placing and Management of Contracts for Building and Civil Engineering Works. HMSO, London.

Berger, P.L. \& Luckmann, T. (1966) The Social Construction of Reality. Doubleday, Garden City, NY.

British Property Federation (1983) Manual of the BPF System for Building Design and Construction. British Property Federation, London.

Construction Industry Research and Information Association (1995) Planning to Build?. Special Publication 113.

Cooper, R., Kagioglou, M., Aouad, G., Hinks, J., Sexton, M. \& Sheath, D. (1998) Development of a generic design and construction process, pp. 205-214. European Conference on Product Data Technology, PDT Days '98, BRE.

Cooper, R.G. (1994) Third-generation new product processes. Fournal of Product Innovation Management, 11, 3-14.

Egan, J. (1998) Rethinking Construction. Department of the Environment, Transport and the Regions, London.

Hughes, W. (1991) Modelling the construction process using plans of work. Construction project modelling and productivity. Proceedings of an International Conference CIB W65, Dubrovnik.

Latham, M. (1994) Constructing the Team. HMSO, London. Linstone, H.A. (1978) The Dephi technique. In: Handbook of Futures Research (ed. J. Fowles), pp. 293-300. Greenwood Press, London.

Luck, R. \& Newcombe, R. (1996) The case for the integration of the project participants activities within a construction project environment. In: The Organization and Management of Construction: Shaping Theory and Practice, vol. 2 (eds D. A. Langford \& A. Retik), pp. 458-470. Spon, London.

Kagioglou, M., Cooper, R., Aouad, G., Hinks, J., Sexton, M. \& Sheath, D. (1998a) A Generic Guide to the Design and Construction Process Protocol. University of Salford, UK.

Kagioglou, M., Cooper, R., Aouad, G., Sexton, M., Hinks, J. \& Sheath, D. (1998b) Cross-industry learning: the development of a generic design and construction process based on stage/gate new product development processes found in the manufacturing industry. In: Engineering Design Conference '98 (eds S. Sivaloganathan \& T. M. M. Shahin), pp. 595-602. 23-25 June, Brunel University.

Koskela, L. (1992) Application of the new production philosophy to construction. Technical Report \#72, Center for Integrated Facility Engineering, Department of Civil Engineering, Stanford University, USA.

Odman, P. (1985) Hermeneutics. In: The International Encyclopaedia of Education (eds T. Husen \& N. T. Postlethwaite), pp. 2162-2169. Pergamon, Oxford.

O'Reilly, J.J.N. (1987) Better briefing means better buildings. Building Research Establishment Report BR 95, B.R.E., Garston, UK.

RIBA (1980) Handbook of Architectural Practice and Management. RIBA, London.

Rosenau, M. (1996) The PDMA Handbook of New Product Development. John Wiley \& Sons, New York.

Sandywell, B. (1975) Problems of Reflexivity and Dialectics in Sociological Inquiry. Routledge and Kegan Paul, London. 
Sanvido, V. (1990) An integrated building process model. CIC Technical Report No. 1, Pennsylvania State University, USA.

Sheath, D., Woolley, H., Hinks, J., Cooper, R. \& Aouad, G. (1996) The development of a Generic Design and Construction Process Protocol for the UK construction industry. Proceedings of InCIT'96, Sydney, Australia.
Simon, E. (1944) The Placing and Management of Building Contract. HMSO, London.

Sommerville, J. \& Stocks, B. (1996) Realising the client's strategic requirements: motivating teams. Proceedings of $\mathrm{CO}-$ $B R A$ '96, University of the West of England.

Walker, A. (1989) Project Management in Construction, 2nd edn. BSP Professional Books, Oxford. 